\title{
Effluent Control in Enterocutaneous Fistula - Experience Of an Enterostomal Therapist
}

\author{
Ida Nirmal, M.Sc (N), Professor, Enterostomal Therapist \\ Department of Surgical Nursing, College of Nursing, CMC, Vellore.
}

\begin{abstract}
Enterocutaneous fistulas represent a challenging situation with respect to wound care and stoma therapy. Large wounds with exposed bowel require the utilization of multiple wound care modalities to provide a method of control and isolation of fistula effluent to allow for maximum wound healing. An understanding of the principles of wound care and the various techniques and materials that are available is of vital importance to enhance patient comfort and recovery as well as facilitate fistula healing. Skin barriers, adhesives, dressings, pouches, and negative pressure dressings are all materials that are available in the armamentarium of the enterostomal therapist. Proper utilization of these items and appropriate modifications to their application requires an intimate knowledge of the characteristics of the fistula being treated. Wound care management is a key element in the overall care and healing of the enterocutaneous fistula.
\end{abstract}

Keywords: Enterocutaneous fistula (ECF ), pouches, negative pressure dressings, skin barriers and wound management

\section{Introduction}

Perhaps one of the most challenging patient care situations that an enterostomal therapist can encounter is the management and treatment of an enterocutaneous fistula. Achieving predictable and effective containment of the fistula effluent often proves to be essential for patients to effectively cope with this rare and frustrating complication. Because each individual patient situation has its own complexities, management requires developing creative and individual plans of care. Utilization of basic pouching concepts, previously reported customized techniques, and networking with enterostomal therapist are invaluable tools to achieving the goals for management of enterocutaneous fistulas.

\section{Definition and causes of ECF}

Enterocutaneous fistula (ECF) is defined as an abnormal, epithelialized connection from the gastrointestinal tract to the skin. The causes of enterocutaneous fistulas can be remembered using the mnemonic FRIEND: Foreign body, Radiation, Inflammation/Infection/Inflammatory bowel disease, Epithelialization, Neoplasm, and Distal obstruction. Fifteen to twenty-five percent of enterocutaneous fistulas arise spontaneously as in, for example, Crohn's disease or cancer. The remainder occurs postoperatively after surgery for diverticulitis or appendicitis; postoperative fistulas can also occur after surgery for inflammatory bowel disease or complications of radiation therapy or if an extensive lysis of adhesions was performed. [1]

III. Classification of ECF [2]

1. Definition of the anatomic course of a fistula is necessary as it may suggest the etiology of the fistula and aid in estimating likelihood of spontaneous closure. Knowledge of fistula anatomy is necessary to plan potential operative strategy towards closure

2. Physiologic classification of fistulas is based on output (in ml per day). High output fistulas (greater than $500 \mathrm{ml}$ per day) are more likely to originate from the small bowel. Low output fistulas (less than $200 \mathrm{ml}$ per day) are more likely to be colonic in origin. Knowledge of the underlying anatomy and physiology help to anticipate and correct fluid and metabolic derangements.

3. The etiology of the fistula may also aid in predicting spontaneous closure rates and mortality

\section{Goals}

Patients with ECF are generally malnourished, have wound infection and coexisting sepsis. Hence ECF is associated with long hospital stays and high morbidity and mortality. In recent years, there has been an improvement in the mortality rates but the current reported figures still remain at 5\% to 20\% [3-4]. Treatment of this catastrophic complication usually involves multifaceted medical and nursing care. An effective ECF wound care management plan should achieve the following goals [5].

1. Protection of perifistular skin integrity

2. Containment of effluent and odor

3. Allow accurate measurement of effluent 
4. Allow mobility and comfort for the patient

5. Decrease cost and time spent in care

\subsection{Protection of perifistular skin Integrity}

Skin protection and prevention of further skin breakdown surrounding the ECF (perifistular skin )are key components in wound care. There are several causes of impaired skin integrity at the site of the ECF. The four most common causes are mechanical trauma, allergic responses, infections, and chemical irritants. The most common chemical irritant is bowel contents. The enzymatic contents of the effluent are many times more detrimental to the skin integrity than the actual volume of the effluent. These digestive secretions exhibit a toxic effect on living tissue and correlates with our clinical observations that fistula effluent does indeed inhibit wound healing [5]. Healing the surrounding skin, preventing further skin breakdown, and minimizing contamination are key components in the wound management of these patients and are of paramount importance.

\subsection{Containment of effluent and odor}

Containment of the effluent can be accomplished with pouching devices, suction devices, dressings or a combination of these management techniques. Dressing material that absorbs and retains caustic secretions is thought to be a major contributor in the delay of healing of ECFs. Therefore, continuous suction devices or stoma application has been advocated as an adjunct to wound care and skin preservation[6]. However, the patient's overall clinical status may play a role in devising the best solution for that individual patient.

Though not commonly addressed, persistent odor from the ECF can be a tremendous source of anxiety and social concern for these patients. Odor control is best controlled with the use of a pouch. Most pouches have an odor-proof film and both internal and external deodorants are available and can help with odor elimination. Deodorants are available in tablet, liquid, or powder forms. Special deodorizers can be placed in the pouch to assist with odor control such as listerin mouth wash

\subsection{Allow accurate measurement of effluent}

Measurement of the fluid and electrolyte balances in these patients is another important goal in wound care of the ECF patient. Leakage around catheters can give an inaccurate reading of actual fluid losses. Dressings can also give an inaccurate reading unless the dressings are weighed on a regular basis. These factors must be taken into account during wound management of ECF patients. Especially with short bowel and proximal ECFs, fluid losses can be up to multiple liters daily. Concomitant electrolyte abnormalities can lead to secondary effects ranging from cardiac arrhythmias to renal failure. Accurate measurement of the effluent is essential to help guide the fluid and nutritional needs in these often sick patients [7].

\subsection{Allow mobility and comfort for the patient}

Skin irritation and discomfort can seriously affect the patient if the wrong management technique is used. Certain pouches or appliances with the use of belts can help minimize and prevent unnecessary patient discomfort, which is a key psychosocial component of wound management in these patients. Ambulatory patients should not be restricted in their recovery and their mobility should not be compromised by a wound management system. Therefore, individualization is an important part in devising the best care for a patient with an ECF.

\subsection{Decrease cost and time spent in care}

Cost-effective medical care is also a principal component in the wound care of patients with ECFs. Attention must be applied not only to the products and materials used for wound care, but labor and time must also be considered. Skin care rituals that consume excessive time and expense without resulting in optimal patient outcomes must be eliminated. Therefore, cost containment is another essential goal in the treatment of ECF patients.

\section{5.Nursing management of ECF}

Management of ECF from the perspective of an enterostomal therapist depends on the materials selected based on the characteristics of the fistula such as output, type of drainage, location, and perifistular skin integrity. Materials include skin barriers; adhesives; dressings, pouches and wound managers; and negative pressure dressings such as vacuum-assisted closure.

\subsection{Skin Barriers}

ECFs can often represent very difficult situations with respect to skin protection. Skin barriers protects the skin by forming physical barrier between effluent and the skin surface. Skin barriers are available as solid wafers, powder, paste, and sealants. (Fig- 1)

DOI: 10.9790/1959-0603010106 $\quad$ www.iosrjournals.org $2 \mid$ Page




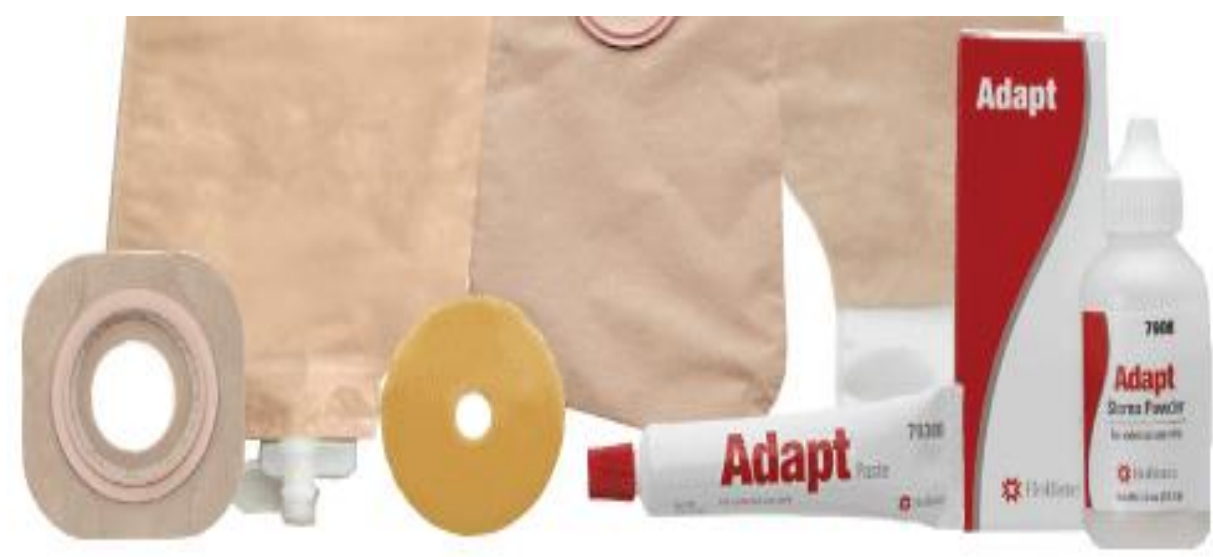

Figure -1

\section{Skin barrier products}

5.1.1 Wafers: Solid wafers are pectin-based products that have their own adhesive surface. This surface melts on contact with the patient's warm skin and creates a seal. Wafers are changed only when they loosen from wound edges or melt out.

5.1.2 Skin barrier powders: Skin barrier powders are used in fistulas with associated skin wounds that are wet and weeping. A thin layer of powder is applied to the denuded skin and allowed to dry before application of the barrier wafer or adhesive surface of a pouch system.

5.1.3 Skin barrier pastes: Used to reinforce the inner edges of solid wafers to protect them from melt out and establish an improved seal. Used to smooth out irregularities in perifistular skin to create a flat surface for wafer application.

5.1.4 Skin sealants: Skin sealants are used to protect the skin surface from adhesives and to create a tacky surface for improved adherence of pouches such as $3 \mathrm{M}$ Cavilon no-sting barrier. Sealants are also used to seal in powders on denuded skin surfaces and facilitate pouch placement.

5.1.5 Zinc oxide: They are are used to waterproof and protect the skin. Again, a generous amount with continuous replacement is necessary because the cream is washed away with discharging enteric effluents.

\subsection{Dressings}

Overlying gauze dressings in combination with skin barriers can be used to effectively contain and manage ECF effluent. However, if fistula output is considered moderate or high or the dressings are being changed more frequently than every 4 hours, a pouching system should be implemented. In this instance, frequent dressing changes become impractical and do not offer the ability to accurately record fistula output. In addition, frequent tape changes associated with dressings can also jeopardize skin integrity especially in the setting of already compromised skin due to effluent-induced irritation.

\subsection{Adhesives}

Adhesives are used to enhance or extend another product's adhesive surface or attach two surfaces when modifying a fistula collection system. Mobile and flaccid skin seems to benefit in particular from adhesive use to improve the seal and increase the wear time of a pouching system. Adhesives are available in three forms: liquid, aerosol, and double-faced adhesive sheet or disc. Liquid and aerosol adhesives contain solvents and must be applied slowly and evaporate before application of any additional products. Sheets of adhesives or doublefaced adhesive discs can be used to create or increase the adhesive surface of a pouch when unusually shaped pouch apertures are required.

\subsection{Pouches:}

The choice of pouch system to be used depends on the characteristics of the fistula being managed. High-output fistulas with very thin liquid effluent are best managed with pouches that have a urinary outlet system to allow for easy drainage and emptying.

Continuous drainage can be achieved with urinary outlet pouches by attaching drainage tubing to the urinary spigot ( Fig -2 ) This can greatly enhance nursing efficiency. 

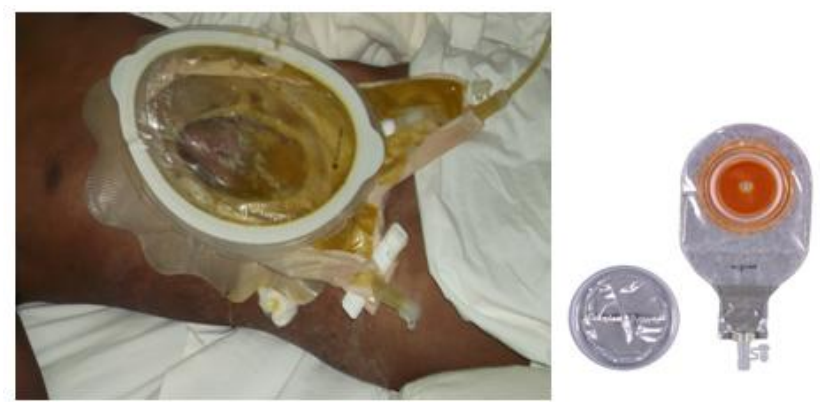

Figure - 2 Urinary outlet pouches

Alternative pouch outlets include fecal outlets with a drainable clip

(Fig - 3) that is appropriate for fistulas with 24-hour output less than $1000 \mathrm{~mL}$ and wide tubular outlets or wound managers

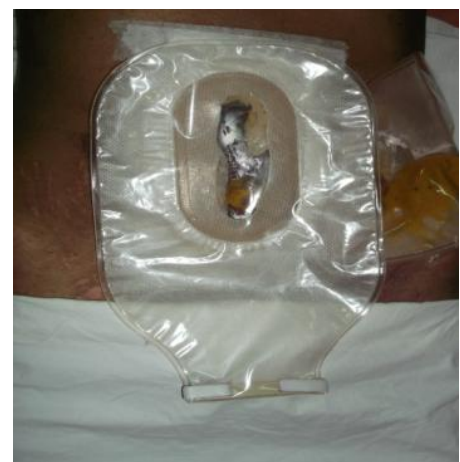

Figure $-\mathbf{3}$ fecal outlet pouches

One piece pouches (Fig- 4) are preferable as they lack an attachment ring and are therefore more pliable for application to irregular skin surfaces. Two-piece pouches (Fig - 5) allow for access to the fistula without removing the entire pouch.

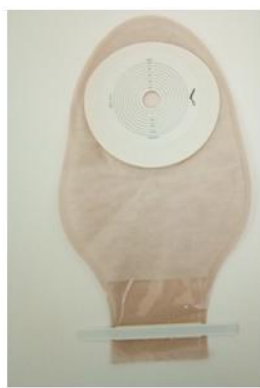

Figure -4

One piece stoma appliance

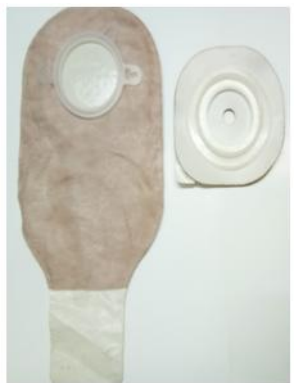

Figures -4

Two- piece stoma appliance

Care must be taken to size the orifice in the adhesive surface appropriately so as to ensure accommodation of the fistula with minimum exposure of the perifistular skin to the effluent (Fig - 5)

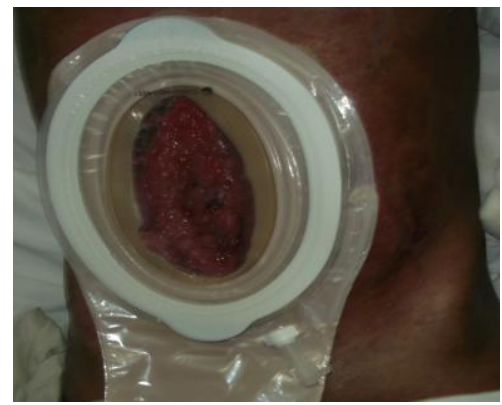

Figure -5 
Sizing the orifice of stoma bag to accommodate fistula

\section{Pouch modifications:}

\subsubsection{Trough Procedure:}

This is useful if the fistula is recessed within a wound that makes routine pouch application fail. The wound is covered with a transparent dressing $(\mathrm{Fig}-6)$ in which a hole is cut in the most dependent portion of the wound over which a pouch is applied (Fig - 7). To enhance adhesion of the transparent dressing and maximize skin protection from effluent, strips of barrier wafer can be cut and applied to the wound edge prior to application of the transparent dressing.

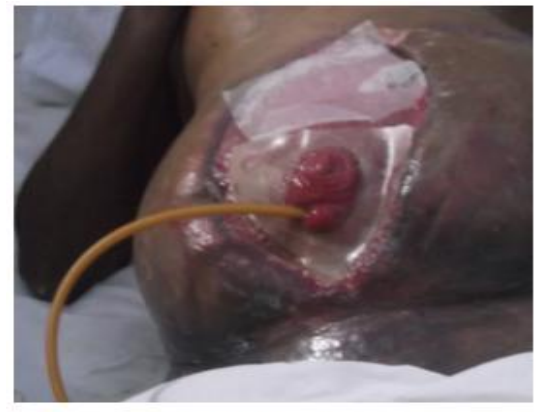

Figure -6

Fistula that is recessed within a wound

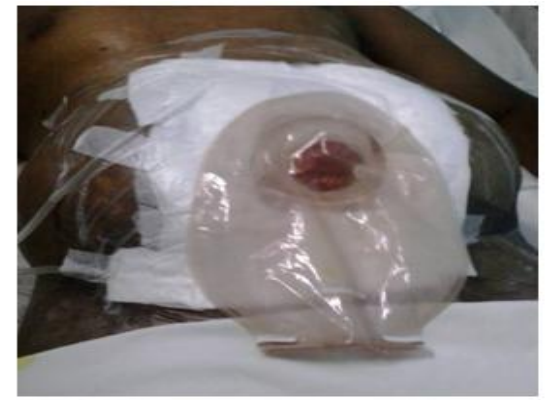

Figures -7

Application of pouch over the transparent dressing

\subsubsection{Bridging Technique}

Complex wounds may occasionally have areas that require different care. This technique can be used to isolate one area of the wound that requires drainage containment from other areas that may require packing or dressing changes. Solid wafer skin barriers cut into small pieces and placed at the bridge location to help facilitate pouch placement. (Figures $-8,9 \& 10$ )

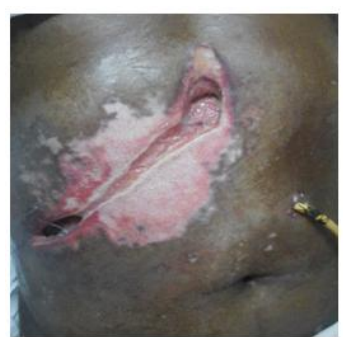

Figure- 8



Figure- 9

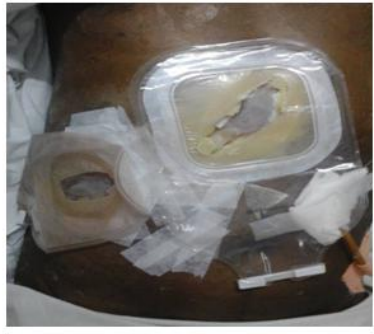

Figure -10

Bridging with skin barrier paste and solid wafers

\subsubsection{Skin Barrier Molds:}

A complex pouch technique that can be used when the fistula orifice is recessed in an open wound or surrounded by numerous irregular skin surfaces. The mold fills (Fig - 11) the wound and creates a flat surface for pouch adherence.

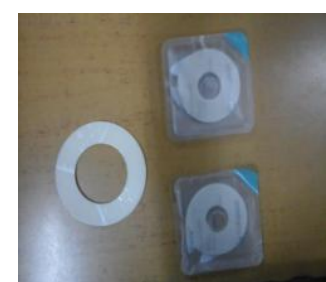

Figure -11 skin barrier molds

\section{6.Topical negative pressure ( TNP )/ Vacuum assisted closure dressing :}

Used extensively in the management of complex wounds (Fig - 12). It enhances wound granulation and wound contracture. Also applied to wounds with ECFs to contain fistula effluent and enhance wound healing. V.A.C. dressings can be tailored to individual wounds (Fig - 13). Different sponges are available for 
use (open pore structure 400-600 microns) that allow for exudate removal and promote granulation formation. In most cases where the V.A.C. dressing was used, it enabled earlier discharge from the hospital and did enhance perifistular wound healing but did not lead to fistula closure. Modifications to this technique have shown great promise where the V.A.C. dressing is applied to the perifistular wound while skin barrier wafers are used to isolate the fistula itself [8].

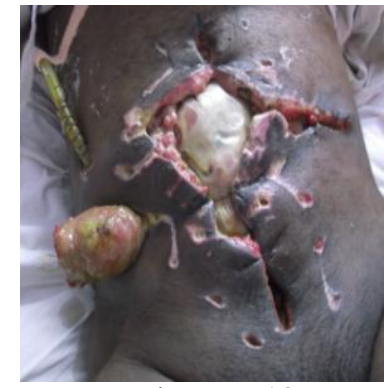

Figure -12

Complex wound

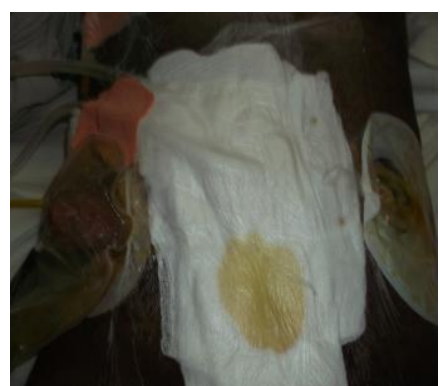

Figure - 13

V.A.C dressing tailored to the complex wound

The fistula is then managed with the pouch applied on top of the V.A.C. dressing, thereby isolating fistula drainage from the remainder of the wound while taking advantage of all the benefits of a negative pressure dressing.

\section{VII.Conclusion}

The wound management of an ECF is very demanding and often a frustrating challenge for an enterostomal therapist. Today, there are many products available to facilitate wound management of the ECF patient. Individualization of a specific wound management system in the ECF patient is very feasible with the multitude of products available once the fistula has been assessed and goals identified. Once wound care is under control through the use of various products and methods discussed, then nutritional, medical and surgical management can proceed for the benefit of the patient.

\section{References}

[1]. Fisher Scott, Enterocutaneous Fistula , Common Surgical Diseases: An Algorithmic Approach to Problem Solving (New York Springer 1998 ) 164-167

[2]. Holzheimer RG, Mannick JA, Surgical Treatment: Evidence-Based and Problem-Orient (Munich: Zuckschwerdt 2001) NCBI Bookshelf. A service of the National Library of Medicine, National Institutes of Health.

[3]. Irrgang S, Bryant R. Management of the enterocutaneous fistula (continuous education credit) J Enterostomal Ther. 1984;11(6):211-228. [PubMed]

[4]. Draus JM Jr, Huss SA, Harty NJ, Cheadle WG, Larson GM. Enterocutaneous fistula: are treatments improving? Surgery. 2006;140:570-576; discussion 576-578. [PubMed]

[5]. Hollington P, Mawdsley J, Lim W, Gabe SM, Forbes A, Windsor AJ. An 11-year experience of enterocutaneous fistula. Br J Surg. 2004;91:1646-1651. [PubMed]

[6]. Beck D. Intestinal fistulas. The Phoenix. 2005;Winter: 58-61.

[7]. Goverman J, Yelon J A, Platz J J, Singson R C, Turcinovic M. The "Fistula VAC," a technique for management of enterocutaneous fistulae arising within the open abdomen: report of 5 cases. J Trauma. 2006;60(2):428-431. discussion 431. [PubMed]

[8]. Haffejee AA. Surgical management of high output enterocutaneous fistulae: a 24-year experience. Curr Opin Clin Nutr Metab Care. 2004;7:309-316. [PubMed]

[9]. Cro C, George K J, Donnelly J, Irwin S T, Gardiner K R. Vacuum assisted closure system in the management of enterocutaneous fistulae. Postgrad Med J. 2002;78(920):364-365. [PMC free article] [PubMed] 